\title{
ON THE GROUP OF ALL HOMEOMORPHISMS OF A MANIFOLD $\left({ }^{1}\right)$
}

\author{
BY \\ GORDON M. FISHER
}

Introduction. It is shown in this paper that the identity component of the group $G(M)$ of all homeomorphisms of a closed manifold of dimension $\leqq 3$ is

(1) simple in the algebraic sense;

(2) equal to the group of deformations of $M$ (i.e., the group of homeomorphisms of $M$ isotopic to the identity homeomorphism);

(3) open in $G(M)$.

The proofs for dimensions 2 and 3 are given separately; the proof for dimension 1, the circle, is not given here, but can be modeled after the proof for dimension 2.

Theorem 9 of this paper (the main tool in obtaining the above results in the case $n=3$ ) is very similar to a recent independent result of Kister appearing elsewhere in this journal (see also [1]).

It is possible to deduce from the above results that the space of homeomorphisms of $M$ is locally arcwise connected. However, since this is a quite special case of more general theorems of Hamstrom and Dyer [2] and Hamstrom [3], we omit the proof.

It is also shown in this paper that if $n \leqq 3$, then the group of deformations of the sphere $S_{n}$ is of index 2 in $G\left(S_{n}\right)$. Furthermore, we find a characterization of homeomorphisms of degree 1 on a closed orientable manifold $M$ of dimension $\leqq 3$ which admits a homeomorphism of degree -1 . Finally, we conclude as a corollary that if $n \leqq 3$, then two homeomorphisms of $S_{n}$ are isotopic if and only if they are homotopic.

These two sets of results are related by the following fact. In showing the results of the first paragraph, we show that if $M$ is a closed manifold of dimension $\leqq 3$, then a homeomorphism $h$ of $M$ is a deformation if and only if $h=h_{1} \cdots h_{k}$ where $h_{i}$ is a homeomorphism of $M$ which is the identity outside some internal closed $n$-cell $F_{i}$ in $M, n=\operatorname{dim} M$ (a closed cell is internal if it lies in an open cell; actually, we do not use all of the internal closed $n$-cells in $M$.) In showing the results of the second paragraph, we show that if $M$ is a closed orientable manifold of dimension $\leqq 3$ which admits a homeomorphism of degree -1 , then a homeomorphism $h$ of $M$ is of degree 1 if and only

Received by the editors December 18, 1959 and, in revised form, February 22, 1960.

(1) This is the author's doctoral dissertation, directed by Professor R. D. Anderson at Louisiana State University, Baton Rouge. The author would like to thank Professor Anderson for his help and encouragement. The author would also like to thank Professor Herman Meyer of the University of Miami (Florida) for various kinds of help through the years. 
if $h=h_{1} \cdots h_{k}$ where $h_{i}$ is a homeomorphism of $M$ which is the identity inside a closed $n$-cell in $M, n=\operatorname{dim} M$ (again we do not use all of the closed $n$-cells in $M$ ).

The history of these problems is, to the best of my knowledge, as follows. In 1914, Tietze showed that the group of indicatrix-preserving homeomorphisms of the 2-sphere $S_{2}$ is of index 2 in the group $G\left(S_{2}\right)$ of homeomorphisms of $S_{2}$ [4]. This was shown again by Kneser in 1926 [5] who also shows that $G\left(S_{2}\right)$ is locally arcwise connected (see [2]), and that these results hold also for $G\left(S_{1}\right)$. In 1928, Baer showed that two homeomorphisms of an orientable closed 2-manifold are homotopic if and only if they are isotopic [6; 7]. In 1934 , Schreier and Ulam $[8 ; 9]$ showed the index 2 result for the arc-component of the identity of $G\left(S_{1}\right)$ and $G\left(S_{2}\right)$, and they showed that the arccomponent of the identity of $G\left(S_{1}\right)$ is simple (using results of Kneser [10] and Poincaré [11]). In 1947, Ulam and von Neumann announced in an ab. stract [12] that the component of the identity of $G\left(S_{2}\right)$ is simple. In 1955 , Fine and Schweigert studied $G(I)$ and $G\left(S_{1}\right)$ (where $I$ is the closed arc) [13]. They found the normal subgroups of $G(I)$, obtained a group-theoretic characterization of $G(I)$, and proved certain theorems on the factoring of homeomorphisms of $I$ and $S_{1}$ into involutions. In 1958, Anderson showed that the groups of all "orientation-preserving" homeomorphisms of $S_{2}$ and $S_{3}$ are simple [14]. Also in 1958, Hamstrom and Dyer showed that the group of all homeomorphisms of a compact 2-manifold is locally contractible [2]. They remark that this generalizes results of Fort [15] and Roberts [16]. In 1959, Hamstrom announced that the space of homeomorphisms of a compact 3manifold is $L C^{n}$ for every $n$ [3]. Roberts observes in [16] that Sanderson has proved that the group of all homeomorphisms of $S_{3}$ is locally arcwise connected.

1. Preliminary remarks on manifolds. Let $X$ denote a topological space, and $A$ a subspace of $X$. The complement, closure, interior, and boundary of $A$ in $X$ are denoted by $X-A, \mathrm{Cl}(A), \operatorname{Int}(A)$, and $\operatorname{Bndy}(A)$; or, when necessary, by $\operatorname{Cl}(A ; X)$, etc. Let $G(X)$ denote the group of all homeomorphisms of $X$, with identity $e$ (or, when necessary, $e_{X}$ ). Let $R^{n}$ denote the $n$-dimensional cartesian space, with the topology induced by the pythagorean metric $d$. For each $a$ in $R^{n}$ and each $r$ in $R=R^{1}$, set

$$
\begin{aligned}
C_{n}(a ; r) & =\left\{x \in R^{n}: d(x, a) \leqq r\right\}, \\
O_{n}(a ; r) & =\left\{x \in R^{n}: d(x, a)<r\right\}, \\
S_{n-1}(a ; r) & =\left\{x \in R^{n}: d(x, a)=r\right\} .
\end{aligned}
$$

Set $I=\{x \in R: 0 \leqq x \leqq 1\}$.

A subspace $C$ of $R^{n}$ is a closed $n$-cell in $R^{n}$ if there exists a homeomorphism $h$ of $C$ onto $C_{n}(0 ; 1)$. Open $n$-cells and $(n-1)$-spheres in $R^{n}$ are defined similarly. A closed $n$-cell $C$ in $R^{n}$ is tame if there exists a homeomorphism $h$ in 
$G\left(R^{n}\right)$ such that $h(C)=C_{n}(0 ; 1)$. Otherwise the cell $C$ is wild. Tame and wild open $n$-cells and $(n-1)$-spheres are defined similarly. Furthermore, tame and wild $m$-cells and $(m-1)$-spheres in $R^{n}$ can be defined for any $m \leqq n$.

It is easily verified that in $R^{1}$, every 0 -sphere and 1 -cell is tame. Schoenflies has shown $[17 ; 18 ; 19]$ that in $R^{2}$ every $m$-cell and $(m-1)$-sphere is tame, $m \leqq 2$. The result of Schoenflies on 1 -spheres in $R^{2}$ can be stated as follows (cf. also the Riemann mapping theorem):

Schoenflies extension THeOREM. Let $S$ and $S^{\prime}$ be 1 -spheres in $R^{2}$, let $h$ be any homeomorphism of $S$ onto $S^{\prime}$, let $B$ and $B^{\prime}$ be the bounded components of $R^{2}-S$ and $R^{2}-S^{\prime}$ (Jordan curve theorem), let $C=S \cup B$ and $C^{\prime}=S^{\prime} \cup B^{\prime}$, and let $A$ be any 2-cell in $R^{2}$ such that $C \cup C^{\prime} \subset \operatorname{Int}(A)$. Then there is a homeomorphism $h^{\prime}$ of $C$ onto $C^{\prime}$ such that $h^{\prime} \mid S=h$, and $h^{\prime}$ can be extended to a homeomorphism $h^{*}$ in $G\left(R^{2}\right)$ such that $h^{*} \mid R^{2}-A$ is the identity.

In $R^{3}$, the situation is quite different. Antoine [20] and Alexander [21] have shown that there are $m$-cells and $(m-1)$-spheres, $1 \leqq m \leqq 3$ (except for 0 -spheres), which are wild in $R^{3}$ (see also Fox and Artin [22]). Nevertheless, a partial substitute in $R^{3}$ for Schoenflies' theorem can be obtained by combining a result of Alexander [23] as proved by Graeub [24] (and Moise [25]), and results of Bing $[26 ; 27 ; 28]$ which were originally obtained using results of Moise [29].

A manifold or $n$-manifold is here a connected separable metric space each point of which has a neighborhood (open set in the metric topology on $M$ ) whose closure in $M$ is homeomorphic to $C_{n}(0 ; 1)$. If $n=0$, connectedness is not required; a 0 -sphere, consisting of two points, is a 0 -manifold. More precisely, it is required that if $x$ is in $M$, then for some neighborhood $U$ of $x$, there is a coordinate homeomorphism $k$ of $C_{n}(0 ; 1)$ onto $\mathrm{Cl}(U)$. If $C$ is a tame closed $n$-cell in $R^{n}$, then there is an $h$ in $G\left(R^{n}\right)$ such that $h(C)=C_{n}(0 ; 1)$, and $k h$ is a homeomorphism of $C$ onto $\mathrm{Cl}(U)$. Any such $k h$ is also called a coordinate homeomorphism.

The core of $M$, denoted by $\operatorname{Core}(M)$, is the space of all points of $M$ which have a neighborhood homeomorphic to $O_{n}(0 ; 1)$. The rim of $M$, denoted by $\operatorname{Rim}(M)$, is the space $M-\operatorname{Core}(M)$. Thus $\operatorname{Core}(M)$ is open in $M$, and $\operatorname{Rim}(M)$ is closed in $M$. (The core and rim of a manifold are traditionally called the interior and boundary; in this paper, the latter words are reserved for the point-set concepts with these names.) A manifold $M$ is closed if it is compact and rimless $(\operatorname{Rim}(M)=\varnothing)$.

Cells and spheres in $M$ are defined as in $R^{n}$. Let $F_{0}$ be a subspace of $M$. A subspace $F$ of $M$ is tame with respect to $F_{0}$ if there is an $h$ in $G(M)$ such that $h(F)=F_{0}$. Otherwise, $F$ is wild with respect to $F_{0}$.

We note, for later use, the following two easily verified facts: (1) if $M$ is an $n$-manifold, then $\operatorname{Rim}(M)$ (which is closed in $M$ ) is nowhere dense in $M$; (2) if $M$ is connected, then so is Core $(M)$. 
2. The smallest nontrivial normal subgroup $G^{0}(M)$. A closed $n$-cell $F$ in an $n$-manifold is internal if there is an open $n$-cell $U=k\left(O_{n}(0 ; 1)\right)$ in $M$ such that, for some $C_{n}(a ; r)$ in $O_{n}(0 ; 1), F=k\left(C_{n}(a ; r)\right)$. Thus such an $F$ lies in Core $(M)$. (It appears to be true that any closed $n$-cell in $\operatorname{Core}(M)$ is internal. but this will not be needed in what follows.)

Let $\mathcal{F}$ denote the set of all internal closed $n$-cells in $M$. Let $E^{0}(M)$ denote the set of all $h$ in $G(M)$ such that, for some $F$ in $F, h$ is the identity on $M-F$; this happens if and only if $h$ is the identity on $M-\operatorname{Int}(F)$. Such an $h$ is the identity outside $F$, or supported on $F$, and $F$ is the support of $h$. If $h$ is in $E^{0}(M)$, say $h$ is supported on $F$ in $\mathcal{F}$, then $h^{-1}$ is also supported on $F$, so $h^{-1}$ is in $E^{0}(M)$. If $f$ is in $G(M)$, then $f h f^{-1}$ is supported on $f(F)$. The homeomorphism $f k$ of $O_{n}(0 ; 1)$ onto $f(U)$ is a coordinate homeomorphism for $f(U)$, and $f k\left(C_{n}(a ; r)\right)=f(F)$, so $f(F)$ is in $F$. Hence $f h f^{-1}$ is in $E^{0}(M)$. Let $G^{0}(M)$ be the subgroup of $G(M)$ generated by $E^{0}(M)$. Since $E^{0}(M)$ is closed under inversion and conjugation, $G^{0}(M)$ is a normal subgroup of $G(M)$, and $h$ is in $G^{0}(M)$ if and only if $h$ can be factored into a finite product, $h=h_{1} \cdots h_{k}$, of homeomorphisms $h_{i}$ of $M$, each supported on some $F_{i}$ in $F$.

In this section it will be shown that $G^{0}(M)$ is the smallest nontrivial $(\neq e)$ normal subgroup of $G(M)$, and that it is simple.

Theorem 1. Let $M$ be an n-manifold. If $F$ is any internal closed $n$-cell in $M$, and $F^{\prime}$ is any closed $n$-cell in $M$, there is a homeomorphism $f$ in $G^{0}(M)$ such that $f(F) \subset F^{\prime}$.

Proof. As we remarked in $\S 1$, Core $(M)$ is connected. The set $F^{\prime}$ of all interiors of cells of $F$ is an open covering of $\operatorname{Core}(M)$. Hence, by a standard theorem of topology, there is a finite collection $V_{1}, \cdots, V_{k}$ of cells from $F^{\prime}$ such that $V_{\boldsymbol{i}} \cap V_{i+1} \neq \varnothing, V_{1}$ meets $\operatorname{Int}(F)$ and $V_{k}$ meets $\operatorname{Core}\left(F^{\prime}\right)$. We may assume $V_{1}=\operatorname{Int}(F)$. Each $\mathrm{Cl}\left(V_{i}\right)$ is in $F$. Hence $\mathrm{Cl}\left(V_{i}\right)=k_{i}\left(C_{i}\right) \subset k_{i}(O)$, where the $k_{i}$ are coordinate homeomorphisms, $C_{i}=C_{n}\left(a_{i} ; r_{1}\right)$, and $O=O_{n}(0 ; 1)$. It is easily seen that $A_{i}=\operatorname{Int}\left(\mathrm{Cl}\left(V_{i}\right) \cap \mathrm{Cl}\left(V_{i+1}\right)\right) \neq \varnothing$. Select $D_{i}=C_{n}\left(b_{i} ; s_{i}\right)$ in $k_{i}^{-1}\left(A_{i}\right)$. There exists an $E=C_{n}(0 ; t), t<1$, such that $D_{i} \cup C_{i} \subset E \subset O$ for every $i$. It is easy to describe a homeomorphism $h_{i}$ of $O$ which takes $C_{i}$ onto $D_{i}$ and is supported on $E$. Define $f_{i}$ by $f_{i}=k_{i} h_{i} k_{i}^{-1}$ on $k_{i}(E)$ and $f_{i}=$ identity on $M-\operatorname{Int}\left(k_{i}(E)\right)$. Then $f_{i}$ is in $E^{0}(M)$ and takes $\mathrm{Cl}\left(V_{i}\right)$ into $A_{i}$. The homeomorphism $f=f_{k-1} \cdots f_{1}$ is in $G^{0}(M)$, and takes $\mathrm{Cl}\left(V_{1}\right)=F$ into $A_{k-1} \subset F^{\prime}$.

The next theorem stems from a theorem of Anderson ([14], Theorem I).

Theorem 2. Let $M$ be an $n$-manifold, and take any $h \neq e$ in $G(M)$. If $f$ is in $G^{0}(M)$, then $f$ is a product of conjugates of $h$ and $h^{-1}$ by homeomorphisms in $G^{0}(M)$.

Proof. As we remarked in $\$ 1, \operatorname{Rim}(M)$ is nowhere dense in $M$. Hence, by the continuity of $h^{-1}$ and the assumption $h \neq e$, there is an $x$ in $\operatorname{Core}(M)$ such that $h^{-1}(x) \neq x$. Using again the continuity of $h^{-1}$ and other elementary facts, 
it follows that there is an open $n$-cell $U=k\left(O_{n}(0 ; 2)\right)$ in Core $(M)$ such that $U \cap h^{-1}(U)=\varnothing$. We will construct in $U$ a pairwise disjoint sequence of closed $n$-cells converging to a point of $U$ and a homeomorphism of $M$ which takes each $n$-cell of the sequence onto the next $n$-cell of the sequence.

Let $A_{0}=C_{n}((3 / 8,0, \cdots, 0) ; 1 / 9)$. Then $A_{0}$ is a closed solid $n$-sphere with center on the $x_{1}$-axis which is caught between the two $(n-1)$-spheres $S_{n-1}(0 ; 1 / 2)$ and $S_{n-1}(0 ; 1 / 4)$; that is, $A_{0}$ is contained in the interior of $C_{n}(0 ; 1 / 2)-\operatorname{Int}\left(C_{n}(0 ; 1 / 4)\right)$. Define a function $r_{1}$ by $r_{1}(x)=x$ on $O_{n}(0 ; 2)$ $-\operatorname{Int}\left(C_{n}(0 ; 1)\right)$ and $r_{1}(x)=d(x, 0) x$ on $C_{n}(0 ; 1)$ (here $R^{n}$ is regarded as a vector space over $R$ ). Set $A_{i+1}=r_{1}\left(A_{i}\right), i \geqq 0$. The sequence $A_{0}, A_{1}, \cdots$ of closed solid $n$-spheres is pairwise disjoint and converges to the origin 0 of $R^{n}$. One verifies that $r_{1}$ is a homeomorphism of $O_{n}(0 ; 2)$.

Set $E=k\left(C_{n}(0 ; 1)\right), B_{i}=k\left(A_{i}\right)$, and $B=U B_{i}$. Then $B_{0}, B_{1}, \cdots$ is a pairwise disjoint sequence of closed $n$-cells in $\operatorname{Int}(E)$, and the homeomorphism defined by $r_{2}=k r_{1} k^{-1}$ on $E$ and $r_{2}=$ identity on $M-\operatorname{Int}(E)$ is in $E^{0}(M)$, and satisfies $B_{i+1}=r_{2}\left(B_{i}\right), i \geqq 0$. It will be convenient to set $r=r_{2}^{-1}$. Then $r$ is in $E^{0}(M)$ and $r\left(B_{i+1}\right)=B_{i}, i \geqq 0$, so $r$ takes each $n$-cell in the sequence $B_{1}, B_{2}, \cdots$ onto the $n$-cell just preceding it in the sequence. Furthermore, $r\left(B_{0}\right)$ misses $B$ completely.

Let $g$ be any homeomorphism of $M$ supported on the cell $B_{0}$. Then $r^{-i} g r^{i}$ is supported on $r^{-i}\left(B_{0}\right)=B_{i}, i \geqq 0$. Define $\phi$ by $\phi\left|B_{i}=r^{-i} g r^{i}\right| B_{i}$ and $\phi=$ identity on $M-\operatorname{Int}(B)$. In particular, $\phi\left|B_{0}=g\right| B_{0}$. One verifies that $\phi$ is in $G(M)$ (because the pairwise disjoint sequence $B_{0}, B_{1}, \cdots$ converges to a point). (It is sometimes helpful to think of $\phi$ as acting like $g$ on each $B_{i}$, although this is only true modulo r.) Since $B \subset E$ and $\phi$ is supported on $B$; $\phi$ is supported on $E$, so $\phi$ is in $E^{0}(M)$.

Consider

$$
\begin{aligned}
w & =\left(r^{-1} \phi^{-1} h^{-1} \phi r\right)\left(r^{-1} h r\right) h^{-1}\left(\phi^{-1} h \phi\right) \\
& =\left(r^{-1} \phi^{-1} h^{-1} \phi h\right) r\left(h^{-1} \phi^{-1} h\right) \phi .
\end{aligned}
$$

The first expression for $w$ shows that $w$ is a product of four conjugates of $h$ and $h^{-1}$. We will show that $w=g$. (It is perhaps interesting to note in the second expression for $w$ that $w$ is a commutator of $r$ and $h^{-1} \phi^{-1} h \phi$, while $h^{-1} \phi^{-1} h \phi$ is in turn a commutator of $h$ and $\phi$.)

Since $\phi$ is supported on $E, h^{-1} \phi^{-1} h$ is supported on $h^{-1}(E)$. Therefore, since $r$ is supported on $E$ and $E \cap h^{-1}(E)=\varnothing, r\left(h^{-1} \phi^{-1} h\right)=\left(h^{-1} \phi^{-1} h\right) r$; that is, $r$ and $h^{-1} \phi^{-1} h$ commute. It follows, after canceling in the second expression for $w$, that $w=r^{-1} \phi^{-1} r \phi$. Since $\phi$ is supported on $B$ and whenever $x$ is not in $B, r(x)$ is also not in $B, w$ is supported on $B$. An easy calculation shows that $w \mid B_{i}$ $=$ identity for $i \geqq 1$. In this connection, note that $\phi\left|B_{i}=r^{-i} g r^{i}\right| B_{i}$ (so $\phi$ "puts $g$ on each $\left.B_{i}{ }^{\prime}\right), r$ takes $B_{i}$ back to $B_{i-1}, \phi^{-1}\left|B_{i-1}=r^{-(i-1)} g^{-1} r^{i-1}\right| B_{i-1}$ (so $\phi^{-1}$ "puts $g^{-1}$ on each $B_{i-1}$ " and "cancels the $g$ which $\phi$ put there"), and $r^{-1}$ takes each $B_{,-1}$ back to $B_{i}$. Thus $w$ is supported on $B_{0}$. On $B_{0}$, the action of $w$ 
is different, and in fact $w\left|B_{0}=g\right| B_{0}$. For, $\phi$ is $g$ on $B_{0}, r$ moves $B_{0}$ onto the set $r\left(B_{0}\right)$ which misses $B, \phi^{-1}$ leaves $r\left(B_{0}\right)$ pointwise fixed since it is supported on $B$, and $r^{-1}$ takes $r\left(B_{0}\right)$ back onto $B_{0}$ (with $g$ "left on it"). Hence, since $w$ and $g$ are both supported on $B_{0}$ and $w\left|B_{0}=g\right| B_{0}, w=g$.

Take any $f$ in $E^{0}(M)$, say $f$ is supported on the internal closed $n$-cell $F$. By Theorem 1, there is a homeomorphism $t$ in $G^{0}(M)$ such that $t(F) \subset B_{0}$. The homeomorphism $t f t^{-1}$ of $M$ is supported on $t(F)$, therefore on $B_{0}$. Hence, as we have just shown, $t f t^{-1}=\left(r^{-1} \phi^{-1} h^{-1} \phi r\right)\left(r^{-1} h r\right) h^{-1}\left(\phi^{-1} h \phi\right)$. Therefore $f=\left(t^{-1} r^{-1} \phi^{-1} h^{-1} \phi r t\right)\left(t^{-1} r^{-1} h r t\right)\left(t^{-1} h^{-1} t\right)\left(t^{-1} \phi^{-1} h \phi t\right)$, so $f$ is a product of four conjugates of $h$ and $h^{-1}$ by homeomorphisms in $G^{0}(M)$. If $f$ is in $G^{0}(M)$, say $f=f_{1} \cdots f_{k}$ where each $f_{i}$ is in $E^{0}(M)$, then each $f_{i}$ is a product of four conjugates of $h$ and $h^{-1}$, so $f$ is a product of $4 k$ conjugates of $h$ and $h^{-1}$, by homeomorphisms in $G^{0}(M)$.

A subgroup $B$ of a group $A$ is normal if $a B=B a$ for every $a$ in $A$ or, what is the same, if for every $a$ in $A$ and $b$ in $B$, the conjugate $a b a^{-1}$ is in $B$. A subgroup $B$ of a group $A$ is nontrivial if $B \neq e$, and proper if $B \neq e$ and $B \neq A$. A group $C$ is simple if it has no proper normal subgroups.

THeOREM 3. Let $M$ be an n-manifold, and let $N$ be the set of all nontrivial normal subgroups of $G(M)$. Then $G^{0}(M)=\bigcap N$, and this group is simple.

Proof. Since $G^{0}(M)$ is a nontrivial normal subgroup of $G(M)$, to obtain the first conclusion it is sufficient to show that if $N$ is any nontrivial normal subgroup of $G(M)$, then $G^{0}(M) \subset N$. Take any such $N$, and any $h \neq e$ in $N$. If $f$ is in $G^{0}(M)$, then, by Theorem $2, f$ is a product of conjugates of $h$ and $h^{-1}$. Hence $f$ is in $N$, so $G^{0}(M) \subset N$.

If $A \neq e$ is a subgroup of $G^{0}(M)$, normal in $G^{0}(M), h \neq e$ is in $A$, and $f$ is in $G^{0}(M)$, then, by Theorem $2, f$ is a product of conjugates of $h$ and $h^{-1}$ by homeomorphisms in $G^{0}(M)$, so $f$ is in $A$. Hence $A=G^{0}(M)$, which means that $G^{0}(M)$ is simple.

Two homeomorphisms $g$ and $h$ in $G(X), X$ any space, are isotopic if there is a family $\left\{H_{t}\right\}, t \in I$, each $H_{t}$ in $G(X)$, such that $H_{0}=g, H_{1}=h$, and the function $H$ from $M \times I$ onto $M$ defined by $H(x, t)=H_{t}(x)$ is continuous; that is, $g$ and $h$ are homotopic, and the homotopy is at each stage a homeomorphism of $X$. An $h$ in $G(X)$ which is isotopic to the identity $e$ is a deformation of $X$. The set of all deformations of $X$ is denoted by $D(X)$.

THEOREM 4. The set $D(X)$ of deformations of a space $X$ forms a normal subgroup of $G(X)$. If $X=M$ is an n-manifold, then every $f$ in the group $G^{0}(M)$ of homeomorphisms of $M$ is a deformation of $M$. (Cf. the theorem of Veblen and Alexander $[30 ; 31]$.)

Proof. The family $\left\{H_{t}\right\}, H_{t}=e$ for all $t$ in $I$, is an isotopy of $e$ and $e$. If $\left\{H_{t}\right\}$ is an isotopy of $h$ and $e$, then $\left\{H_{1-t} h^{-1}\right\}$ is an isotopy of $h^{-1}$ and $e$. If $\left\{H_{t}^{\prime}\right\}$ is an isotopy of $g$ and $e$, then $\left\{g H_{2 t}: 0 \leqq t \leqq 1 / 2\right\} \cup\left\{H_{2 t-1}^{\prime}: 1 / 2 \leqq t \leqq 1\right\}$ is an isotopy of $g h$ and $e$. If $p$ is in $G(M)$, then $\left\{p H_{t} p^{-1}\right\}$ is an isotopy of 
$p h p^{-1}$ and $e$. Hence $D(X)$ is a normal subgroup of $G(X)$. The second statement of the theorem now follows from Theorem 3.

3. Deformations of a 1 -sphere or closed 2-manifold. It is easily verified that every 1-manifold is triangulable. It is known that every 2 -manifold and 3-manifold is triangulable (Rado [32], Gawehn [33], Moise [29], Bing [26; $28])$. These facts will be used throughout the rest of this paper.

If $X$ is a compact space, $Y$ is a metric space with metric $d$, and $f$ and $g$ are (continuous) maps of $X$ into $Y$, then

$$
p(f, g)=\operatorname{lub}\{d(f(x), g(x): x \in X\}
$$

defines the Fréchet metric in the set of all maps of $X$ into $Y$. If $X=Y$, then the set $G(X)$ of homeomorphisms of $X$ is a topological group in the topology induced by $p$ (see, e.g., [3]).

We will need the following theorem, which can be thought of as an extension of the theorem of Schoenflies quoted in $\$ 1$. A proof of this theorem can be found in [2, Lemma 2], or can be obtained using conformal mapping theory. The referee has informed me that Roberts has also given a proof of this theorem (mentioned by him in [16]).

THEOREM 5. Let $d A$ and $d B$ be two circles in $R^{2}$ with center at the origin 0 bounding the closed discs $A$ and $B$, where $A \subset B$. For every $r>0$, there is an $s>0$ such that, given any homeomorphism $h$ of $A$ into $B$ for which $d(x, h(x))<s$ for every $x$ in $A$ (i.e., $\left.p\left(h, e_{B}\right)<s\right)$, there is a homeomorphism $h^{*}$ in $G(B)$ such that $h^{*}\left|A=h, h^{*}\right| d B=i d e n t i t y$, and $d\left(x, h^{*}(x)\right)<r$ for every $x$ in $B$ (i.e., $\left.p\left(h^{*}, e_{B}\right)<r\right)$.

THEOREM 6. Let $M$ be a closed 2-manifold or the 1-sphere. There is a number $s>0$ such that, if $h$ is in $G(M)$ and $p(h, e)<s$, then $h$ is in $G^{0}(M)$. That is, the subgroup $G^{0}(M)$ of the topological group $G(M)$ contains a neighborhood of the identity e, open in $G(M)$.

Proof. A proof for $M$ a closed 2-manifold will be given; the case $M=S_{1}$ can be handled analogously.

Let $T$ be a euclidean 2-complex which triangulates $M$, and $\phi$ a homeomorphism of $T^{*}$ onto $M$. Since $M$ is a closed 2-manifold, so is the polyhedron $T^{*}$. We will first prove the theorem for $T^{*}$.

Let $T^{\prime}$ denote the subdivision of $T$ obtained by joining each barycenter of a 2-simplex of $T$ to the three vertices of that simplex. Let $T^{(2)}$ denote the second barycentric subdivision of $T$. Let $U_{1}, \cdots, U_{k}$ be all those subspaces of $T^{*}$ which are the union of two closed 2-simplexes of $T^{\prime}$ which have a common edge in $T$. Since $T$ is finite, there are only a finite number of such sets. We may assume that each $U_{i}$ and each 2-simplex of $T$ is internal (for example, we could take a covering of $T^{*}$ by open 2-cells and a complex $K$ of all whose simplexes are of diameter less than a Lebesgue number of this covering, and then start from the beginning with $K$ ). Let $V$ be the union of the 
closed stars in $T^{(2)}$ of the vertices of $T$ (the closed star $S_{i}^{(2)}$ in $T_{i}^{(2)}$ of a vertex $v_{i}$ of $T$ is the union of all closed simplexes of $T^{(2)}$ which contain $v_{i}$ ). Let $r>0$ be the least distance between any two components of $T_{1}^{\prime *}-V$, where $T_{1}^{\prime}$ is the 1-skeleton of $T^{\prime}$ (the set of closed 1-simplexes in $T^{\prime}$ ).

We assert that any $f$ in $G\left(T^{*}\right)$ satisfying $f \mid V=$ identity and $p(f, e)<r / 2$. is in $G^{0}\left(T^{*}\right)$. In fact, let $S$ be a 2 -simplex of $T^{\prime}$. Let $g_{S}$ be defined on $\operatorname{Bndy}(S)$ by $g_{S}=f$ on the edge of $S$ which is in $T$, and $g_{S}=$ identity on the other two edges of $S$; this is possible since $f \mid V=$ identity. Then $g_{S}$ is a homeomorphism whose domain is $\operatorname{Bndy}(S)$. Since $p(f, e)<r / 2$, the range of $g_{S}$ is contained in the $U_{i}$ containing $S$. By the Schoenflies theorem quoted in Chapter $\mathrm{I}, g_{S}$ can be extended to a homeomorphism whose domain is $S$ and whose range is contained in the $U_{i}$ containing $S$; denote the extension of $g_{S}$ again by $g_{S}$. The homeomorphisms $g_{S}, S$ in $T$, can be pieced together in an obvious way to obtain a homeomorphism $g^{\prime}$ of $T^{*}$ (there are $2 k$ of the homeomorphisms $g_{S}$, where $k$ is the number of edges in $T$ ). The homeomorphism $g^{\prime}$ is the identity on $\operatorname{Bndy}\left(U_{i}\right)$ for each $i$. Hence $g^{\prime}=g_{1}^{\prime} \cdots g_{k}^{\prime}$, where $g_{i}^{\prime} \mid U_{i}=g^{\prime}$. Therefore, since each $U_{i}$ is an internal closed 2-cell in $T^{*}, g$ is in $G^{0}\left(T^{*}\right)$.

Now $f=g^{\prime} g^{\prime-1} f$, and since $g^{\prime}=f$ on the boundary of every 2 -simplex in $T$, we have that $g^{-1} f$ is the identity on the boundary of every 2 -simplex $S$ in $T$. Hence, as in the last paragraph, $g^{\prime-1} f=f_{1} \cdots f_{k^{\prime}}$, where $f_{i}\left|S=g^{-1} f\right| S$; the integer $k^{\prime}$ is the number of 2 -simplexes in $T$. Since each 2 -simplex $S$ in $T$ is internal, $g^{-1} f$ is in $G^{0}\left(T^{*}\right)$. Therefore, since $g^{\prime}$ is in $G^{0}\left(T^{*}\right)$, so is $g^{\prime} g^{\prime-1} f=f$.

We now assert that there is a number $s>0$ such that, if $h$ is in $G\left(T^{*}\right)$ and $p(h, e)<s$, then there is a $g$ in $G^{0}\left(T^{*}\right)$ such that $g^{-1} h \mid V=$ identity, and $p\left(g^{-1} h, e\right)<r / 2$. The theorem (for $\left.T^{*}\right)$ follows from this assertion, since if $h$ is in $G\left(T^{*}\right), p(h, e)<s$, and $g$ in $G^{0}\left(T^{*}\right)$ is of the kind just described, then $h=g g^{-1} h$ and, by what we proved above (taking $\left.f=g^{-1} h\right), g^{-1} h$ is in $G^{0}\left(T^{*}\right)$; hence $h$ is in $G^{0}\left(T^{*}\right)$.

Let $v_{1}, \cdots, v_{m}$ be the vertices of $T$ (by the Euler-Poincare formula, $m=\chi\left(T^{*}\right)+k-k^{\prime}$, where $\chi\left(T^{*}\right)$ is the Euler characteristic of $\left.T\right)$. Let $S_{i}^{(1)}$ be the closed star of $v_{i}$ in the first barycentric subdivision $T^{(1)}$ of $T$, and let $S_{i}^{(2)}$ be the closed star of $v_{i}$ in the second barycentric subdivision. $T^{(2)}$. Let $A$ and $B$ be the closed discs of Theorem 5 , and let $\phi_{i}$ be any homeomorphism of $B$ onto $S_{i}^{(1)}$ which takes $A$ onto $S_{i}^{(2)}$ (that such a $\phi_{i}$ exists is a consequence of the Schoenflies exiinsion theorem, see, e.g., [19]). Let $d$ be the pythagorean metric in $R^{2}$, and let $d^{\prime}$ be the pythagorean metric in $T^{*}$ (subspace of $R^{5}$ ). Let $r^{\prime}>0$ be so small that if $d(x, y)<r^{\prime}$, then $d^{\prime}\left(\phi_{i}(x), \phi_{i}(y)\right)<r / 4$ for all $x$ and $y$ in $B$ and all $i$; such an $r^{\prime}$ exists because of the uniform continuity of the $\phi_{i}$ on $B$. Let $s^{\prime}>0$ be so small that if $f$ is a homeomorphism of $A$ into $B$ and $p\left(f, e_{B}\right)<s^{\prime}$, then $f$ has an extension $f^{*}$ to $B$ such that $f^{*}=$ identity on $\operatorname{Bndy}(B)$ and $p\left(f^{*}, e_{B}\right)<r^{\prime}$; such an $s^{\prime}$ exists by Theorem 5 . Let $s>0$ be so small that $s<r / 4$ and, if $d^{\prime}\left(x^{\prime}, y^{\prime}\right)<s$, then $d\left(\phi_{i}^{-1}\left(x^{\prime}\right), \phi_{i}^{-1}\left(y^{\prime}\right)\right)<s^{\prime}$ for all $x^{\prime}$ and $y^{\prime}$ in $S_{i}^{(1)}$; such an $s$ exists by the uniform continuity of the $\phi_{i}^{-1}$ on $S_{i}^{(1)}$.

Take any $h$ in $G\left(T^{*}\right)$ such that $p^{\prime}\left(h, e_{T^{*}}\right)<s$. If $x$ is in $A$, then 
$d^{\prime}\left(h \phi_{i}(x), \phi_{i}(x)\right)<s$ for all $i$. Hence $d\left(\phi_{i}^{-1} h \phi_{i}(x), \phi_{i}^{-1} \phi_{i}(x)\right)=d\left(\phi_{i}^{-1} h \phi_{i}(x), x\right)$ $<s^{\prime}$, so $p\left(\phi_{i}^{-1} h \phi_{i} \mid A, e_{B}\right)<s^{\prime}$ for all $i$. Therefore $\phi_{i}^{-1} h \phi_{i} \mid A$ has an extension $h_{i}^{*}$ to $B$ such that $h_{i}^{*} \mid \operatorname{Bndy}(B)=$ identity and $p\left(h_{i}^{*}, e_{B}\right)<r^{\prime}$ for all $i$. Since $d^{\prime}\left(h_{i}^{*} \phi_{i}^{-1}\left(x^{\prime}\right), \phi_{i}^{-1}\left(x^{\prime}\right)\right)<r^{\prime}$ for all $x^{\prime}$ in $\phi(B)=S_{i}^{(1)}$ and all $i$, we have $d^{\prime}\left(\phi_{i} h_{i}^{*} \phi_{i}^{-1}\left(x^{\prime}\right), \phi_{i} \phi_{i}^{-1}\left(x^{\prime}\right)\right)=d^{\prime}\left(\phi_{i} h_{i}^{*} \phi_{i}^{-1}\left(x^{\prime}\right), x^{\prime}\right)<r / 4$ for all $x^{\prime}$ in $S_{i}^{(1)}$ and all $i$. Define $h_{i}$ by $h_{i}\left(x^{\prime}\right)=\left(\phi_{i} h_{i}^{*} \phi_{i}^{-1}\right)\left(x^{\prime}\right)$ for $x^{\prime}$ in $S_{i}^{(1)}$ and $h_{i}=$ identity otherwise; this is possible because $h_{i}^{*} \mid \operatorname{Bndy}(B)=$ identity. Then $p^{\prime}\left(h_{i}, e_{T^{*}}\right)<r / 4$, $h_{i}\left|S_{i}^{(2)}=h\right| S_{i}^{(2)}$, and $h_{i}$ is in $G^{0}\left(T^{*}\right)$.

Let $g=h_{1} \cdots h_{m}$. Then $g$ is in $G^{0}\left(T^{*}\right)$ and, because the supports $S_{i}^{(1)}$ of the $h_{i}$ meet only in edges on which they are the identity, $p^{\prime}\left(g, e_{T^{*}}\right)<r / 4$. Hence $p^{\prime}\left(g^{-1} h, e_{T^{*}}\right) \leqq p^{\prime}\left(g^{-1}, e_{T^{*}}\right)+p^{\prime}\left(h, e_{T^{*}}\right)=p^{\prime}\left(g, e_{T^{*}}\right)+p^{\prime}\left(h, e_{T^{*}}\right)<r / 4+r / 4$ $=r / 2$. Moreover $g\left|S_{i}^{(2)}=h_{i}\right| S_{i}^{(2)}=h \mid S_{i}^{(2)}$. Therefore $g^{-1} h=$ identity on $S_{i}^{(2)}, i$ $=1, \cdots, m$, so $g^{-1} h=$ identity on $V=U S_{i}^{(2)}$.

This proves the theorem for $T^{*}$. To prove the theorem for $M$, let $d^{\prime \prime}$ be the given metric in $M$, and let $t>0$ be so small that if $x$ and $y$ are in $M$ and $d^{\prime \prime}(x, y)<t$, then $d^{\prime}\left(\phi^{-1}(x), \phi^{-1}(y)\right)<s$; such a $t$ exists by the uniform continuity of the triangulation homeomorphism $\phi$ of $T^{*}$ onto $M$. Take any $h$ in $G(M)$ such that $p^{\prime \prime}\left(h, e_{M}\right)<t$. Then $p^{\prime \prime}(h \phi, \phi)<t$, so $p^{\prime}\left(\phi^{-1} h \phi, e_{T^{*}}\right)<s$. Therefore $\phi^{-1} h \phi$ is in $\left.G^{0} T^{*}\right)$, so $\phi^{-1} h \phi=f_{1} \cdots f_{k}, f_{i}$ supported on $F_{i} \subset T^{*}$. Hence

$$
h=\phi f_{1} \phi^{-1} \phi f_{2} \phi^{-1} \cdots \phi f_{k} \phi^{-1},
$$

$\phi f_{i} \phi^{-1}$ supported on $\phi\left(F_{i}\right) \subset M$. Hence $h$ is in $G^{0}(M)$.

In connection with the following theorem, we note that the identity component $C_{e}(M)$ of the topological group $G(M)$ is the largest connected subspace of $G(M)$ containing $e$; in any topological group, the identity component is a closed normal (topological) subgroup. We also note that it is easily verified that $D(M)$ is the arc-component of the identity in the topological group $G(M)$; that is, $D(M)$ is the set of all $h$ in $G(M)$ which can be joined to $e$ by an arc lying in $G(M)$.

TheOREM 7. Let $M$ be a closed 2-manifold or the 1-sphere. The identity component $C_{e}(M)$ of the group $G(M)$ of all homeomorphisms of $M$ is simple, open in $G(M)$, and equal to (1) the group $G^{0}(M)$ of all $h$ in $G(M)$ such that $h=h_{1} \cdots h_{k}$ where $h_{i}$ is supported on an internal closed 2-cell $F_{i}$ in $M$ (or 1-cell if $M=S_{1}$ ), and (2) the group $D(M)$ of all deformations of $M$ (all $h$ in $G(M)$ isotopic to the identity $e)$.

Proof. By Theorem $6, G^{0}(M)$ is an open subgroup of $G(M)$ (since the neighborhood of $e$ can be translated to any point of $\left.G^{0}(M)\right)$. Therefore $G^{0}(M)$ is a closed subgroup of $G(M)$.

As we remarked above, $C_{e}(M)$ is a closed normal subgroup of $G(M)$, and by its definition it is connected. By Theorem $3, G^{0}(M) \subset C_{e}(M)$. Since $G^{0}(M)$ is open and closed in $G(M)$ and contained in $C_{e}(M)$, it is open and closed in $C_{e}(M)$. Hence $G^{0}(M)=C_{e}(M)$. Since $G^{0}(M)$ is open in $G(M)$, so is $C_{e}(M)$. Since $D(M)$ is the arc-component of $e$, it is connected and contains $e$, so 
$D(M) \subset C_{e}(M)$. By Theorem 4, $G^{0}(M) \subset D(M)$. Therefore $G^{0}(M)=D(M)$ $=C_{0}(M)$.

4. Deformations of a closed 3-manifold. The terms locally tame, locally polyhedral, and piecewise linear used in this section are defined in [26].

In place of the Schoenflies extension theorem of $\S 1$, and Theorem 5 of $\S 3$, the following three theorems will be used:

Alexander's extension theorem [23] (as proved by Graeub [24, Theorem $1, \S 5]$ ). Let $S$ and $S^{\prime}$ be polyhedral 2-spheres in a triangulated 3-manifold $M$ which are contained in the interior of a closed polyhedral 3-cell $A$ in $M$, let $B$ and $B^{\prime}$ be the rimless components of $A-S$ and $A-S^{\prime}$ (Jordan-Brouwer theorem), and let $C=S \cup B$ and $C^{\prime}=S^{\prime} \cup B^{\prime}$. There is a piecewise linear homomorphism $h^{\prime}$ of $C$ onto $C^{\prime}$ such that $h^{\prime} \mid S=h$, and $h^{\prime}$ can be extended to a homeomorphism $h^{*}$ in $G(M)$ such that $h^{*} \mid M-A$ is the identity.

Note. Graeub only shows that $A$ can be taken to be a 3-simplex. However, if $A$ is polyhedral, then $\operatorname{Int}(A)$ can be taken piecewise linearly onto $R^{3}$, a 3-simplex containing the images of $C$ and $C^{\prime}$ chosen, Graeub's version of Alexander's theorem applied, and then the whole taken back to $\operatorname{Int}(A)$.

Bing'S EXTENSION THEOREM [26]. Let $M$ be a triangulated 3-manifold, $C$ a closed subspace of $M, K$ a locally tame closed subspace of $M$ such that $K$ is locally polyhedral at each point of $K \cap C$, and $\phi$ a positive continuous function on $M-C$. There is a homeomorphism $f$ in $G(M)$ such that $f(K)$ is a polyhedron, $f$ is the identity on $C$, and $d(x, f(x))<\phi(x)$ for every $x$ in $M-C$.

SANDERSON'S EXTENSION THEOREM [34]. Let L be a closed polyhedral 2manifold in a closed triangulated 3-manifold $M$, let $U$ be an open neighborhood of $L$, and take any $s>0$. There is an $r>0$ such that if $h$ is a piecewise linear homeomorphism of $L$ into $M$ and $p(h, e)<r$, then there is a piecewise linear homeomorphism $g$ in $G(M)$ such that $g|L=h| L, g$ is the identity on $M-U$, and $p(g, e)<s$.

Note. Sanderson proves there is a simplicial $s$-isotopy which takes $h(L)$ pointwise onto $L$ and is the identity on $M-U$. If $f$ is the end-stage of such an isotopy, $f h(x)=x$ for all $x$ in $L$. We take $g=f^{-1}$.

Theorem 8. Let $M$ be a closed 3-manifold. There is a number $s>0$ such that, if $h$ is in $G(M)$ and $p(h, e)<s$, then $h$ is in $G^{0}(M)$. That is, the subgroup $G^{0}(M)$ of the topological group $G(M)$ contains a neighborhood of the identity e, open in $G(M)$.

Let $T_{i}$ denote the $i$-skeleton of a triangulation $T$ of $M$. We will consider tubular neighborhoods of $T_{1}^{*}$, as defined by Moise in [35].

We will deal first with homeomorphisms of $M$ which are the identity inside of a tubular neighborhood $N$ of $T_{1}^{*}$. By the definition of tubular neighbor- 
hood, $N$ is polyhedral in $M$ (with respect to $T$ ). Let $T^{\prime}$ be the subdivision of $T$ obtained by taking the join of each barycenter of a 3-simplex of $T$ with the vertices of that simplex. It follows from the definition of tubular neighborhood that the components of $T_{2}^{*}-N$ are the interiors of a disjoint collection of closed polyhedral 2-cells in $M$, each lying in a face of some tetrahedron of $T$. Let $t_{1}>0$ be the least distance between components of $T_{2}^{*}-N$, let $t_{2}>0$ be the least distance between a component of $T_{2}{ }^{*}-N$ and a 2 -simplex of $T_{2}^{\prime}$ not in $T_{2}$, and set $t=\min \left\{t_{1}, t_{2}\right\}$. Let $h$ be any homeomorphism in $G(M)$ such that $p(h, e)<t / 2$ and $h \mid N=$ identity. We will show that $h$ is in $G^{0}(M)$.

Consider, on each 2-simplex $s^{2}$ in $T_{2}$, the homeomorphism $h \mid s^{2}$. Because $h \mid N$ is the identity and $p(h, e)<t / 2, h\left(s^{2}\right)$ is contained in the union $U=s^{3} \cup t^{3}$ of two simplexes $s^{3}$ and $t^{3}$ of $T_{3}^{\prime}$ which have $s^{2}$ as a common face. By Bing's extension theorem, there is a homeomorphism $f$ in $G(M)$ such that $f$ is the identity on all 2-simplexes of $T_{2}^{\prime}$ except those in $T_{2}, p(f, e)<t / 2$, and $f h\left(s^{2}\right)$ is a polyhedral 2-cell (take $K$ to be $h\left(T_{2}^{*}\right)$, and $C$ to be the union of the 2simplexes of $T_{2}^{\prime}$ not in $T_{2}$; then $\left.K \cap C=\varnothing\right)$. Since $p(f h, e)<t, f h\left(s^{2}\right)$ is contained in $U$. Now $f h\left(s^{2}\right)$ together with the three faces of $s^{3}$ besides $s^{2}$, and $f h\left(s^{2}\right)$ together with the three faces of $t^{3}$ besides $s^{2}$, form two polyhedral 2spheres $S$ and $S^{\prime}$. Define a homeomorphism $h_{s}$ on Bndy $\left(s^{3}\right)$ by $h_{s}\left|s^{2}=f h\right| s^{2}$, and $h_{s}=$ identity on the other three sides of $s^{3}$; define similarly an $h_{t}$ for $t^{3}$. By Alexander's extension theorem, there is an extension $\phi_{8}$ of $h_{8}$ to $s^{3}$, and an extension $\phi_{t}$ of $h_{t}$ to $t^{3}$. The homeomorphisms $\phi_{s}$ and $\phi_{t}$ agree on $s^{2}$, and can be pieced together to give a homeomorphism $\phi_{U}$ of $U$ which is the identity on Bndy $(U)$, and $f h$ on $s^{2}$. The homeomorphisms $\phi_{U}$ (one for each $s^{2}$ in $T_{2}$ ) can be pieced together in an obvious way to give a homeomorphism $\phi$ of $T^{*}$ such that $\phi$ is the identity on each $\operatorname{Bndy}(U)$, and $f h$ on each $s^{2}$ in $T_{2}$. The homeomorphism $f^{-1} \phi$ is also the identity on each $\operatorname{Bndy}(U)$, and is $h$ on each $s^{2}$ in $T_{2}$. Consider $h=f^{-1} \phi \phi^{-1} f h$. Since $f^{-1} \phi$ is the identity on each $\operatorname{Bndy}(U)$, it can be factored into a finite number of homeomorphisms, each the identity outside a $U$. We may assume that $U$ is an internal closed 3-cell in $M$ (by starting out with a triangulation $T$ of sufficiently small mesh). Therefore $f^{-1} \phi$ is in $G^{0}(M)$. Since $\phi^{-1} f$ is $h^{-1}$ on each $s^{2}, \phi^{-1} f h$ is the identity on each $s^{2}$. Hence $\phi^{-1} f h$ can be factored into a finite number of homeomorphisms, each supported on an $s^{3}$ in $T_{3}$. We may assume that each $s^{3}$ is internal. Then $\phi^{-1} f h$ is in $G^{0}(M)$. Therefore $f^{-1} \phi \phi^{-1} f h=h$ is in $G^{0}(M)$.

We will now deal with homeomorphisms which are the identity outside a tubular neighborhood $N_{1}$ of $T_{1}^{*}$ lying in the interior of a tubular neighborhood $N_{2}$ of $T_{1}^{*}$. On each 1 -simplex $s^{1}$ in $T_{1}$, select three inner points $x, y, z$ (in the cylindrical part of $N_{2}$ ), and three polyhedral discs $D_{x}, D_{y}, D_{z}$ which are disjoint cross-sections of (the cylindrical part of) $N_{2}$; they are to contain, respectively, $x, y, z$. Assume that $y$ is between $x$ and $z$ on $s^{1}$, and call $D_{y}$ the middle disc for $s^{1}$, and $D_{x}, D_{z}$ the end discs for $s^{1}$. We assume that this has been done in such a way that to each edge in $T_{1}$ there have been assigned ex- 
actly three discs. Let $u_{1}>0$ be the least distance, for any $s^{1}$ in $T_{1}$, between a middle disc of an $s^{1}$ and its two end discs. Let $u_{2}>0$ be the distance from $\operatorname{Bndy}\left(N_{1}\right)$ to $\operatorname{Bndy}\left(N_{2}\right)$. Let $u=\min \left\{u_{1}, u_{2}\right\}$, and take any homeomorphism $h$ in $G(M)$ such that $h$ is the identity outside $N_{1}$ and $p(h, e)<u / 2$. We will show that $h$ is in $G^{0}(M)$.

Each two end discs $D_{x}, D_{z}$ of an $s^{1}$ in $T_{1}$ determine in $N_{2}$ a polyhedral 3-cell consisting of $D_{x}, D_{z}$, and that component of $N_{2}-\left(D_{x} \cup D_{z}\right)$ which contains the point $y$ of $s^{1}$. Denote one of these cells by $V$. The middle disc $D_{y}$ splits $V$ into two smaller polyhedral 3-cells $W$ and $W^{\prime}$. Bndy $(W)$ consists, say, of $D_{x}, D_{y}$, and that part of $\operatorname{Bndy}(V)$ between $D_{x}$ and $D_{y}$, and $\operatorname{Bndy}\left(W^{\prime}\right)$ consists of $D_{y}, D_{z}$, and the rest of $\operatorname{Bndy}(V)$. By Bing's extension theorem, there is an $f$ in $G(M)$ such that $f$ is the identity outside each $V, p(f, e)<u / 2$, and $f h\left(D_{y}\right)$ is a polyhedral disc for every $D_{y}$ (take $K$ to be the union of the sets $h\left(D_{y}\right)$ and $C$ to be $\left.\operatorname{Bndy}\left(N_{2}\right)\right)$. Define a homeomorphism $h_{W}$ on each $\operatorname{Bndy}(W)$ by setting $h_{W}=$ identity except on $D_{y}$, where $h_{W}$ is $f h \mid \operatorname{Bndy}(W)$; define similarly an $h_{W^{\prime}}$ for each Bndy $\left(W^{\prime}\right)$. Each $h_{W}$ is then a homeomorphism of a polyhedral 2-sphere onto another such, so by Alexander's extension theorem, there is a $\phi_{W}$ taking the 3-cell $W$ bounded by the first 2-sphere onto the 3-cell bounded by the second; and similarly there is a $\phi_{W^{\prime}}$ for $W^{\prime}$. Each $\phi_{W}$ and $\phi_{W^{\prime}}$ can be pieced together to give a homeomorphism of $V$ which is the identity on $\operatorname{Bndy}(V)$, and agrees with $f h$ on $D_{y}$. The homeomorphisms so obtained (one for each $s^{1}$ in $T_{1}$ ) can be pieced together to give a homeomorphism $\phi$ in $G(M)$ which is the identity outside the union $U$ of the sets $V$, and is $f h$ on each $D_{y}$. The homeomorphism $f^{-1} \phi$ is also the identity outside $U$, and it is $h$ on each $D_{y}$. Consider $h=f^{-1} \phi \phi^{-1} f h$. Since $f^{-1} \phi$ is the identity outside $U$, it can be factored into a finite product of homeomorphisms, each supported on some one of the 3-cells $V$; hence $f^{-1} \phi$ is in $G^{0}(M)$. Since $\phi^{-1} f$ is $h^{-1}$ on each $D_{y}, \phi^{-1} f h$ is the identity on each $D_{y}$. Consider the sets $P$ which are obtained by running out from a vertex $v$ of $T$, along each edge of $T$ which meets this vertex, until we come to the $D_{y}$ corresponding to this edge, and then taking the union $D$ of these sets $D_{y}$ together with that part of $N_{2}-D$ which contains $v$. It follows from the definition of tubular neighborhood that the sets $P$ are 3 -cells. Now, since $\phi^{-1} f$ is the identity outside $U$, it is the identity outside $N_{2}$ (since $U \subset N_{2}$ ), and $h$ is the identity outside $N_{1}$ by hypothesis. Therefore, since $N_{1} \subset N_{2}, h$ is the identity outside $N_{2}$, so $\phi^{-1} f h$ is the identity outside $N_{2}$. Furthermore, since $f^{-1} \phi$ is $h$ on each $D_{x}$, $\phi^{-1} f$ is $h^{-1}$ on each $D_{x}$, so $\phi^{-1} f h$ is the identity on each $D_{x}$. Thus $\phi^{-1} f h$ can be factored into a finite product of homeomorphisms each supported on some one of the 3-cells $P$. Therefore $\phi^{-1} f h$ is in $G^{0}(M)$. Therefore $f^{-1} \phi \phi^{-1} f h=h$ is in $G^{0}(M)$.

Now let $N_{1}, N_{2}$ and $N_{3}$ be three tubular neighborhoods of $T_{1}^{*}$ such that $N_{1} \subset \operatorname{Int}\left(N_{2}\right)$ and $N_{2} \subset \operatorname{Int}\left(N_{3}\right)$. Let $t>0$ be as in the second paragraph of the proof (using now components of $T_{2}^{\prime}-N_{1}$ ), and let $u>0$ be the number of the last paragraph, determined by discs in $N_{3}$. Let $w=\min \{t / 6, u / 6\}$. By Sanderson's extension theorem, there is an $r$ such that, if $g$ is any piecewise 
linear homeomorphism of $\operatorname{Bndy}\left(N_{1}\right)$ into $M$ such that $p(g, e)<r$, then there is a piecewise linear $\phi$ in $G(M)$ such that $\phi\left|\operatorname{Bndy}\left(N_{1}\right)=g\right| \operatorname{Bndy}\left(N_{1}\right), \phi$ is the identity outside $N_{2}$, and $p(\phi, e)<w$ (take $U$ to be $\operatorname{Int}\left(N_{2}\right)$, and $L$ to be $\operatorname{Bndy}\left(N_{1}\right)$; that $L$ is a closed 2-manifold follows from the definition of tubular neighborhood and the fact that $M$ is closed). Set $s=\min \{w, r / 2\}$. Take any $h$ in $G(M)$ such that $p(h, e)<s$. We will show that $h$ is in $G^{0}(M)$.

By Bing's extension theorem, there is an $f$ in $G(M)$ such that $f h\left(\operatorname{Bndy}\left(N_{1}\right)\right)$ is a polyhedron, $f$ is the identity outside $N_{2}$, and $p(f, e)<s$ (take $K$ to be $h\left(\operatorname{Bndy}\left(N_{1}\right)\right)$ and $C$ to be $\operatorname{Bndy}\left(N_{2}\right)$; then $\left.K \cap C=\varnothing\right)$. Since $p(f h, e)<r$, and $f h$ is a piecewise linear homeomorphism of $\operatorname{Bndy}\left(N_{1}\right)$ into $M$, there is a piecewise linear $\phi$ such that $\phi\left|\operatorname{Bndy}\left(N_{1}\right)=f h\right| \operatorname{Bndy}\left(N_{1}\right), \phi$ is the identity outside $N_{2}$, and $p(\phi, e)<w$. Consider $h=f^{-1} \phi \phi^{-1} f h$. Since $f^{-1} \phi$ is the identity outside $N_{2}, N_{2} \subset \operatorname{Int}\left(N_{3}\right)$, and $p\left(f^{-1} \phi, e\right)<u / 6+u / 6<u / 2, f^{-1} \phi$ is in $G^{0}(M)$, as we showed above. Also $\phi^{-1} f h$ is the identity on Bndy $\left(N_{1}\right)$. Hence $\phi^{-1} f h$ can be factored into two homeomorphisms, $\phi^{-1} f h=a b$, where $a$ is the identity outside $N_{1}$ and $a\left|N_{1}=\phi^{-1} f h\right| N_{1}$, and $b$ is the identity inside $N_{1}$ and $b \mid M-N_{1}$ $=\phi^{-1} f h \mid M-N_{1}$. We have $p\left(\phi^{-1} f h, e\right)<\min \{t / 2, u / 2\}$, so $p(a, e)$ and $p(b, e)$ are also less than either $t / 2$ or $u / 2$. Hence, as we showed above, $a$ and $b$ are in $G^{0}(M)$. Thus $\phi^{-1} f h$ is in $G^{0}(M)$, and therefore so is $f^{-1} \phi \phi^{-1} f h=h$.

This completes the proof of Theorem 8.

Theorem 9. Let $M$ be a closed 3-manifold. The identity component $C_{0}(M)$ of the group $G(M)$ of all homeomorphisms of $M$ is simple, open in $G(M)$, and equal to (1) the group $G^{0}(M)$ of all $h$ in $G(M)$ such that $h=h_{1} \cdots h_{k}$ where $h_{i}$ is supported on an internal closed 3-cell in $M$, and (2) the group $D(M)$ of all deformations of $M$ (all $h$ in $G(M)$ isotopic to the identity $e$ ).

Proof. Same as Theorem 7, using Theorem 8 instead of Theorem 6.

5. The group $G\left(S_{n}\right), n \leqq 3$. Let $M$ be an $n$-manifold, and let $E^{I}(M)$ denote the set of all $h$ in $G(M)$ such that $h$ is the identity inside some closed $n$-cell in $M$ (not necessarily internal). Let $G^{I}(M)$ be the subgroup of $G(M)$ generated by $E^{I}(M)$. Just as in $\S 2$, one verifies that $G^{I}(M)$ is the set of all $h$ in $G(M)$ such that $h=h_{1} \cdots h_{k}$ for some $h_{i}$ in $G(M)$ such that $h_{i}$ is the identity inside some closed $n$-cell $F_{i}$ in $M$.

The following lemma is a consequence of the Alexander and Bing extension theorems quoted in $\$ 4$.

TheOREM 10. Let $M$ be a manifold, $\operatorname{dim} M \leqq 3$. There is an internal closed $n$-cell $F_{0}$ in $M, n=\operatorname{dim} M$, such that, for any $h$ in $G(M)$, there is an $f$ in $G^{0}(M)$ such that $f\left(F_{0}\right)=h\left(F_{0}\right)$ (setwise).

Proof. The cases $n=0$ and $n=1$ are elementary. If $n=2$, the theorem is true for any internal closed 2-cell in $M$; this follows from the Schoenflies extension theorem of $\$ 1$, together with a chain argument similar to that in Theorem 1.

Let $\operatorname{dim} M=3$, and let $M$ be triangulated by the locally finite complex $T$. 
Let $F_{0}$ be an internal closed 3 -simplex in some barycentric subdivision $T^{(i)}$ of $T$ (the open 3 -cell in which $F_{0}$ lies need not be polyhedral). Take an $h$ in $G(M)$ and let $s$ and $s^{\prime}$ be closed 3-simplexes in the barycentric subdivision $T^{(i+2)}$ of $T$ such that $s \subset \operatorname{Int}\left(F_{0}\right)$ and $s^{\prime} \subset \operatorname{Int}\left(h\left(F_{0}\right)\right)$. Set $T^{\prime}=T^{(i+2)}$. Since $T$ is strongly connected, so is $T^{\prime}$, and there is a chain $s_{1}=s, s_{2}, \cdots, s_{r}=s^{\prime}$ of closed 3-simplexes from $s$ to $s^{\prime}$ (such that $s_{i} \cap s_{i+1}$ is a common 2-face of $s_{i}$ and $\left.s_{i+1}\right)$. In the second barycentric subdivision $T^{\prime(2)}$ of $T^{\prime}$, let $S_{1}, \cdots, S_{2 r-1}$ be the closed stars in $T^{\prime(2)}$ of the barycenters of the 3 -simplexes $s_{i}$ together with the closed stars in $T^{\prime(2)}$ of the barycenters of the 2-simplexes $s_{i} \cap s_{i+1}$. Each $S_{i}$ is a closed polyhedral 3-cell, and we can assume that $S_{1}, \cdots, S_{2 r-1}$ are ordered in such a way that each $S_{i} \cup S_{i+1}$ is a closed polyhedral 3-cell and each $S_{i} \cap S_{i+1}$ is a closed polyhedral 2-cell. In each $S_{i}$, choose a closed 3-simplex $t_{i}$ such that $t_{i} \subset \operatorname{Int}\left(S_{i}\right)$. It is not difficult to describe a homeomorphism $g_{i}^{\prime}$ of $S_{i} \cup S_{i+1}$ onto itself which takes $t_{i}$ onto $t_{i+1}$ and is supported on $S_{i} \cup S_{i+1}$ (or one can invoke Alexander's extension theorem). Each $g_{i}^{\prime}$ can be extended to a $g_{i}$ in $G^{0}(M)$ by defining $g_{i} \mid S_{i} \cup S_{i+1}=g_{i}^{\prime}$ and $g_{i}=$ identity outside Int $\left(S_{i} \cup S_{i+1}\right)$. The $g=g_{2 r-2} \cdots g_{1}$ is in $G^{0}(M)$ and $g\left(t_{1}\right)=t_{2 r-1}$, where $t_{1} \subset \operatorname{Int}\left(F_{0}\right), t_{2 r-1} \subset \operatorname{Int}\left(h\left(F_{0}\right)\right)$. Set $t_{1}=t, t_{2 r-1}=t^{\prime}$.

Since the closed 3 -simplex $F_{0}$ is internal, say $F_{0}$ is contained in the open 3-cell $U$, there is a closed 3-simplex $F_{0}^{\prime}$ (not a simplex of a subdivision of $T^{\prime}$, but a union of simplexes of a subdivision of $T^{\prime}$, or $F_{0} \cup\left(\operatorname{Bndy}\left(F_{0}\right) \times I\right)$, where $I$ is a sufficiently short interval) such that $F_{0} \subset \operatorname{Int}\left(F_{0}^{\prime}\right) \subset F_{0}^{\prime} \subset U$, and there is a homeomorphism $r^{\prime}$ which takes $F_{0}$ onto $t$ and is supported on $F_{0}^{\prime}$, hence can be extended to an $r$ in $G^{0}(M)$ such that $r\left(F_{0}\right)=t$.

Since $h\left(F_{0}\right)$ is internal, $h\left(F_{0}\right) \subset h(U)$, there is a closed 3-cell $E^{\prime}$ such that $h\left(F_{0}\right) \subset \operatorname{Int}\left(E^{\prime}\right) \subset E^{\prime} \subset h(U)$. For example, in $k^{-1} h^{-1}(h(U))=O_{3}(0 ; 1)$, where $k$ is a coordinate homeomorphism for $U$, there is a $C_{3}(0 ; t)$ containing $k^{-1} h^{-1}\left(h\left(F_{0}\right)\right)$ in its interior; take $E^{\prime}=h k\left(C_{3}(0 ; t)\right)$. By a theorem of Bing (Theorem 1 of [27] or Theorem 5 of [28]), there is a polyhedral 3-cell $E$ such that $h\left(F_{0}\right) \subset \operatorname{Int}(E) \subset E \subset h(U)$.

By Bing's extension theorem, there is a homeomorphism $p$ in $G(M)$ such that $p\left(h\left(F_{0}\right)\right)=P$, where $P$ is a polyhedral 3-cell in $M, P \subset \operatorname{Int}(E)$, and $p$ is supported on $E$ (so $g$ is in $G^{0}(M)$ ). By Alexander's extension theorem, there is a homeomorphism $q^{\prime}$ of $t^{\prime}$ onto $P$ which is supported on $E$, and can be extended to $q$ in $G^{0}(M)$ such that $q\left(t^{\prime}\right)=P$. Hence $f=p^{-1} q g r$ is in $G^{0}(M)$, and we have $f\left(F_{0}\right)=h\left(F_{0}\right)$.

An $F_{0}$ satisfying the conditions of Theorem 9 is called a pivot cell in $M$. Let $M$ be a manifold, $\operatorname{dim} M \leqq 3$, let $F_{0}$ be a pivot cell in $M$, and let $T\left(F_{0}\right)$ be the set of all cells in $M$ tame with respect to $F_{0}$ (i.e., $F$ is in $T\left(F_{0}\right)$ if and only if there is a $w$ in $G(M)$ such that $F=w\left(F_{0}\right)$ ). For each $F$ in $T\left(F_{0}\right)$, let $P(F)$ denote the set of all $h$ in $G(M)$ such that, for some $f$ in $G^{0}(M), f \mid F$ $=h \mid F$. For each $F$ in $T\left(F_{0}\right)$, let $Q(F)$ denote the set of all $h$ in $G(M)$ such that, for some $f$ in $G^{0}(M), f(F)=h(F)$ and $f^{-1} h \mid B$ is in $G^{0}(B)$, where $B=$ Bndy $F$. (The Brouwer invariance theorem implies that $f^{-1} h$ takes $B$ onto itself.) 
A clearer view of the geometric meaning of $P(F)$ and $Q(F)$ can be obtained by applying Theorems 7 and 9 , and replacing the groups $G^{0}(M)$ and $G^{0}(B)$ by the deformation groups $D(M)$ and $D(B)$. Thus $h$ is in $P(F)$ if and only if the $n$-cell $h(F)(n=\operatorname{dim} M)$ can be "slid back" by an $f^{-1}$ pointwise onto $F$ (i.e., $f^{-1} h(x)=x$ for all $x$ in $F$ ); and $h$ is in $Q(F)$ if and only if the $n$-cell $h(F)$ can be "slid back" setwise by an $f^{-1}$ onto $F$, and $f^{-1} h \mid B$ is a deformation of the $(n-1)$-sphere bounding $F$.

THEOREM 11. Let $M$ be a manifold, $\operatorname{dim} M \leqq 3$, and let $F_{0}$ be a pivot cell in $M$. For each $F$ in $T\left(F_{0}\right), P(F)$ is a normal subgroup of $G(M)$. For each $F$ in $T\left(F_{0}\right), P(F)=P\left(F_{0}\right)$.

Proof. Take any $F$ in $T\left(F_{0}\right)$ and any $h$ in $P(F)$. By definition, there is an $f$ in $G^{0}(M)$ such that $f|F=h| F$. Hence $f^{-1} h \mid F=$ identity. Since $G^{0}(M)$ is normal in $G(M), h^{-1} f^{-1} h$ is in $G^{0}(M)$. Since $h^{-1} f^{-1} h\left|F=h^{-1}\right| F, h^{-1}$ is in $P(F)$. Take $h, g$ in $P(F)$. Then, as we have just shown, $h^{-1}, g^{-1}$ are in $P(F)$. Hence there are $p$ and $q$ in $G^{0}(M)$ such that $p\left|F=h^{-1}\right| F, q\left|F=g^{-1}\right| F$. Since $G^{0}(M)$ is normal in $G(M), h\left(\left(g q^{-1} g^{-1}\right) p^{-1}\right) h^{-1}$ is in $G^{0}(M)$. Hence, since ( $h g)\left(q^{-1} g^{-1}\right)\left(p^{-1} h^{-1}\right)|F=h g| F, h g$ is in $P(M)$. Take any $k$ in $G(M)$. Since $F$ is in $T\left(F_{0}\right)$, there is a $w$ in $G(M)$ such that $F=w\left(F_{0}\right)$. By Theorem 10, there are $r$ and $s$ in $G^{0}(M)$ such that $r\left(F_{0}\right)=w\left(F_{0}\right)=F$, and $s\left(F_{0}\right)=k w\left(F_{0}\right)=k(F)$. Set $t=s r^{-1}$. Then $t$ is in $G^{0}(M)$ and $t(F)=k(F)$. Since $G^{0}(M)$ is normal in $G(M), \quad k^{-1}\left(\left(h t h^{-1}\right) f t^{-1}\right) k$ is in $G^{0}(M)$. Since $\left(k^{-1} h k\right)\left(k^{-1} t\right)\left(h^{-1} f\right)\left(t^{-1} k\right) \mid F$ $=k^{-1} h k \mid F$ (because $h^{-1} \mid F=$ identity), $k^{-1} h k$ is in $P(F)$. This proves that $P(F)$ is a normal subgroup of $G(M)$.

Take $h$ in $P\left(F_{0}\right)$ and $f$ in $G^{0}(M)$ such that $f\left|F_{0}=h\right| F_{0}$. Since $F$ is in $T\left(F_{0}\right)$, there is a $w$ in $G(M)$ such that $F=w\left(F_{0}\right)$. By Theorem 10 , there is a $g$ in $G^{0}(M)$ such that $g\left(F_{0}\right)=w\left(F_{0}\right)=F$. We have $g h g^{-1}\left|F=g f g^{-1}\right| F$. Therefore, since $g f g^{-1}$ is in $G^{0}(M)$, we have that $g h g^{-1}$ is in $P(F)$. Since, as we showed above, $P(F)$ is a normal subgroup of $G(M)$, we have that $g^{-1}\left(g h g^{-1}\right) g=h$ is in $P(F)$. This shows that $P\left(F_{0}\right) \subset P(F)$. Since the argument is symmetric in $F_{0}$ and $F$, we have $P(F)=P\left(F_{0}\right)$.

TheOREM 12. Let $M$ be a manifold, $\operatorname{dim} M \leqq 3$, and let $F_{0}$ be a pivot cell in $M$. For any $F$ in $T\left(F_{0}\right), P(F)=G^{I}(M)$. Hence an $h$ in $G(M)$ is in $G^{I}(M)$, so that $h=h_{1} \cdots h_{k}, h_{i}$ the identity inside some closed $n$-cell $F_{i}$ in $M(n=\operatorname{dim} M)$, if and only if for any $n$-cell $F$ in $M$ tame with respect to $F_{0}$, there is a deformation $f$ of $M$ such that $f(x)=h(x)$ for every $x$ in $F$.

Proof. Take $h$ in $E^{I}(M)$, say $h$ is the identity inside the closed cell $E$. By Theorem 1 , there is an $f$ in $G^{0}(M)$ such that $f\left(F_{0}\right) \subset E$. Then $h$ is the identity inside $f\left(F_{0}\right)$. Hence there is a homeomorphism in $G^{0}(M)$, namely the identity $e$, such that $e\left|f\left(F_{0}\right)=h\right| f\left(F_{0}\right)$. Hence $h$ is in $P\left(f\left(F_{0}\right)\right)$. Now $f\left(F_{0}\right)$ is in $T\left(F_{0}\right)$. Therefore, by Theorem $11, h$ is in $P\left(F_{0}\right)$. Hence, since $P\left(F_{0}\right)$ is a group by Theorem 11, the group $G^{I}(M)$ generated by $E^{I}(M)$ is contained in $P\left(F_{0}\right)$.

Take $h$ in $P\left(F_{0}\right)$ and $f$ in $G^{0}(M)$ such that $f\left|F_{0}=h\right| F_{0}$, so that $f^{-1} h \mid F_{0}$ 
=identity. Since $G^{I}(M)$ is normal in $G(M), G^{0}(M) \subset G^{I}(M)$ by Theorem 3 . Hence $f$ is in $G^{I}(M)$. Also $f^{-1} h$ is in $G^{I}(M)$ (even $E^{I}(M)$ ), since $f^{-1} h \mid F_{0}$ =identity. Therefore $h=f f^{-1} h$ is in $G^{I}(M)$. Thus $P\left(F_{0}\right) \subset G^{I}(M)$.

By the previous two paragraphs, $P\left(F_{0}\right)=G^{I}(M)$. Therefore, by Theorem 11, $P(F)=G^{I}(M)$ for every $F$ in $T\left(F_{0}\right)$.

The following lemma will be needed later; the group $Q\left(F_{0}\right)$ was defined just before Theorem 11.

THEOREM 13. Let $M$ be a manifold, $\operatorname{dim} M \leqq 3$, and let $F_{0}$ be a pivot cell in $M$. For every $F$ in $T\left(F_{0}\right), P(F)=Q\left(F_{0}\right)$.

Proof. Take $h$ in $P\left(F_{0}\right)$ and $f$ in $G^{0}(M)$ such that $f\left|F_{0}=h\right| F_{0}$. Then $f^{-1} h \mid B_{0}$ $=$ identity, where $B_{0}=$ Bndy $F_{0}$, so $h$ is in $Q\left(F_{0}\right)$. Thus $P\left(F_{0}\right) \subset Q\left(F_{0}\right)$.

Take $h$ in $Q\left(F_{0}\right)$ and $f$ in $G^{0}(M)$ such that $f\left(F_{0}\right)=h\left(F_{0}\right)$ and $f^{-1} h \mid B_{0}$ is in $G^{0}\left(B_{0}\right)$. By Theorem $4, f^{-1} h \mid B_{0}$ is in $D\left(B_{0}\right)$. Hence there is a family $\left\{H_{t}\right\}$ $C G\left(B_{0}\right)$ such that $H_{0}=f^{-1} h\left|B_{0}, H_{1}=e\right| B_{0}$, and $H$ from $B_{0} \times I$ onto $B_{0}$ defined by $H(x, t)=H_{t}(x)$ is continuous. Since $F_{0}$ is internal, there is a $U$ such that $F_{0}=k\left(C_{n}\left(0 ; r_{i}\right) \subset U=k\left(O_{n}(0 ; 1)\right)\right.$ for some coordinate homeomorphism $k$ (where $n=\operatorname{dim} M)$. Set $C=C_{n}\left(0 ; r_{i}\right)$, and take $C^{\prime}=C_{n}\left(0 ; r^{\prime}\right)$ such that $C^{\prime} \subset O_{n}(0 ; 1)$ and $C \subset \operatorname{Int}\left(C^{\prime}\right)$. Fiber the (generalized) annulus $A=C^{\prime}-C$ by the closed arcs obtained by taking the intersection of $A$ with each straight line through the origin of $R^{n}$. Also fiber $A$ by the $(n-1)$-spheres $S_{n-1}(0 ; s)$, $r \leqq s \leqq r^{\prime}$. Define a homeomorphism $\phi$ of $A$ as follows. If $x$ is in $S_{n-1}(0 ; s)$, run along the unique arc fiber through $x$ to the point $y$ on $k^{-1}\left(B_{0}\right)=S_{n-1}(0 ; r)$ lying on this arc fiber (an endpoint), move to $k^{-1} H_{u} k(y)$ where $u=s-r / r^{\prime}-r$, run back along the unique arc fiber of which $k^{-1} H_{u} k(y)$ is an endpoint to the unique point $\phi(x)$ in which this arc fiber meets $S_{n-1}(0 ; s)$. Define a $g$ in $G^{0}(M)$ by $g\left|k\left(C^{\prime}\right)-\operatorname{Int}\left(F_{0}\right)=k \phi k^{-1}, g\right| \cdot F_{0}=f^{-1} h\left|F_{0}, g\right| M-\operatorname{Int}\left(k\left(C^{\prime}\right)\right)=$ identity. This definition is consistent, since $g\left|B_{0}=k \phi k^{-1}\right| B_{0}=k k^{-1} H_{0} k k^{-1}\left|B_{0}=H_{0}\right| B_{0}$ $=f^{-1} h \mid B_{0}$, and setting $B=\operatorname{Bndy}\left(k\left(C^{\prime}\right)\right), \quad g\left|B=k \phi k^{-1}\right| B=k k^{-1} H_{1} k k^{-1} \mid B$ $=H_{1} \mid B$ =identity. We have $f g\left|F_{0}=h\right| F_{0}$. Therefore, since $f g$ is in $G^{0}(M), h$ is in $P\left(F_{0}\right)$. Thus $Q\left(F_{0}\right) \subset P\left(F_{0}\right)=Q\left(F_{0}\right)$. The theorem now follows from Theorem 11.

Let $M$ be an orientable closed $n$-manifold. In the sequel, when we speak of a homeomorphism $h$ of degree 1 or -1 of $M$, we refer to the original definition of Brouwer [36]. We will use this concept only in connection with orientable closed manifolds of dimension $\leqq 3$ and the $n$-sphere, all of which are triangulable; hence the original definition in terms of simplicial approximations can be used. When we say that such a manifold is orientable, we mean this in the sense of the theory of simplicial complexes. These concepts can, of course, also be introduced using homology theory, but we will find it more convenient to use the original definitions.

We recall the following facts about the set $B(M)$ of all homeomorphisms on $M$ of degree 1 ( $M$ as in the last paragraph), to be used below. (1) $B(M)$ is a normal subgroup of $G(M)$. This follows from the fact that if $f$ and $g$ are any 
two maps of $M$ into itself, then degree $(f g)=\operatorname{degree}(g f)=\operatorname{degree}(f) \cdot \operatorname{degree}(g)$. (2) For any $n, S_{n}$ admits a homeomorphism of degree -1 . Namely, regard $S_{n}$ as the boundary of an $(n+1)$-simplex, and consider a simplicial homeomorphism which interchanges exactly two vertices. Hence $B\left(S_{n}\right)$ is a proper normal subgroup of $G\left(S_{n}\right)$. (3) Since a homeomorphism of $M$ is either of degree 1 or -1 (see, e.g., [37]), $B(M)$ is of index 2 in $G(M)$. (A subgroup $B$ of a group $A$ is of index 2 in $A$ if there are exactly two left (right) cosets in $A / B$. If $B$ is normal, the "left (right)" can be omitted.)

We note also the following facts about subgroups of index 2 to be used below. (1) $B$ is of index 2 in $A$ if and only if there is an $r$ in $A$ but not in $B$ such that, for every $h$ in $A$ but not in $B, h^{-1} r$ is in $B$; (2) if $B$ is of index 2 in $A$, then there is no subgroup of $A$ properly larger than $B$ and smaller than $A$.

Theorem 14. For any $n, G^{0}\left(S_{n}\right)=G^{I}\left(S_{n}\right)$, and this group is a simple proper normal subgroup of $G\left(S_{n}\right)$. If $n \leqq 3$, the index of $G^{0}\left(S_{n}\right)$ in $G\left(S_{n}\right)$ is 2 . Hence, for $n \leqq 3, G\left(S_{n}\right)$ has exactly the one proper normal subgroup $G^{0}\left(S_{n}\right)=G^{I}\left(S_{n}\right)$.

Proof. By Theorem $3, G^{0}\left(S_{n}\right) \subset G^{I}\left(S_{n}\right)$, since $G^{I}\left(S_{n}\right)$ is normal in $G\left(S_{n}\right)$. If $h$ is in $E^{I}\left(S_{n}\right)$, say $h$ is the identity in the closed $n$-cell $F$, then there is a closed $n$-simplex $s$ in $F$, and $S_{n}-\operatorname{Int}(s)$ is an internal closed $n$-cell in $S_{n}$. Since $h$ is the identity inside $F, h$ is the identity outside $S_{n}-\operatorname{Int}(s)$. Hence $h$ is in $E^{0}\left(S_{n}\right)$. Therefore $G^{0}\left(S_{n}\right)=G^{I}\left(S_{n}\right)$, and this group is simple by Theorem 3 . By Theorem $3, G^{0}\left(S_{n}\right) \subset B\left(S_{n}\right)$. Since $B\left(S_{n}\right) \neq G\left(S_{n}\right)$ (in fact (2) about $B\left(S_{n}\right)$ ), $G^{0}\left(S_{n}\right)$ is proper (clearly, $G^{0}\left(S_{n}\right) \neq e$ ).

To show that $G^{0}\left(S_{n}\right)$ is of index 2 in $G\left(S_{n}\right)$ for $n \leqq 3$, we proceed by induction. The assertion is true for the 0 -sphere $S_{0}$. For, there are only two homeomorphisms of $S_{0}$, the identity $e$, and the homeomorphism $r$ which interchanges the two points of $S_{0}$. Since $r$ is not in $E^{0}\left(S_{0}\right), E^{0}\left(S_{0}\right)=e$; hence $G^{0}\left(S_{0}\right)=e$. Therefore $G\left(S_{0}\right) / G^{0}\left(S_{0}\right)$ has exactly two cosets, $\{e\}$ and $\{r\}$.

Suppose the assertion is true for $n-1$, where $1 \leqq n \leqq 3$. Choose a pivot cell $F_{0}$ in $S_{n}$ (Theorem 10), and set $B_{0}=$ Bndy $F_{0}$. Since $G^{0}\left(S_{n}\right) \subset B\left(S_{n}\right)$ $\neq G\left(S_{n}\right)$, there is a homeomorphism $r$ of $S_{n}$ not in $G^{0}\left(S_{n}\right)$. We will show that for any $h$ in $G\left(S_{n}\right)$ but not in $G^{0}\left(S_{n}\right), h^{-1} r$ is in $G^{0}\left(S_{n}\right)$. By fact (1) about groups of index 2 noted above, this will complete the proof.

Since $r$ is not in the group $G^{0}\left(S_{n}\right)$, neither is $r^{-1}$. By Theorem 10, there are $f, g$ and $k$ in $G^{0}\left(S_{n}\right)$ such that $f\left(F_{0}\right)=r^{-1}\left(F_{0}\right), g\left(F_{0}\right)=h\left(F_{0}\right)$, and $k\left(F_{0}\right)$ $=h^{-1} r\left(F_{0}\right)$. By the first part of this theorem, together with Theorems 12 and $13, G^{0}\left(S_{n}\right)=G^{I}\left(S_{n}\right)=Q\left(F_{0}\right)$. Hence, since $r^{-1}$ and $h$ are not in $Q\left(F_{0}\right), f^{-1} r^{-1} \mid B_{0}$ and $g^{-1} h \mid B_{0}$ are not in $G^{0}\left(B_{0}\right)$. Now $B_{0}$ is an $(n-1)$-sphere. Hence, by the induction hypothesis, $G^{0}\left(B_{0}\right)$ is of index 2 in $G\left(B_{0}\right)$. Therefore $\left(f^{-1} r^{-1} \mid B_{0}\right)\left(g^{-1} h \mid B_{0}\right)$ is in $G^{0}\left(B_{0}\right)$. Now $k\left(F_{0}\right)=h^{-1} r\left(F_{0}\right)=h^{-1} r f^{-1} r^{-1}\left(F_{0}\right)$ $=h^{-1} r f^{-1} r^{-1} g^{-1} h\left(F_{0}\right)$, since $f^{-1} r^{-1}\left(F_{0}\right)=F_{0}=g^{-1} h\left(F_{0}\right)$. Moreover, $h^{-1}\left(\left(r f^{-1} r^{-1}\right) g^{-1}\right) h$ is in the normal subgroup $G^{0}\left(S_{n}\right)$ of $G\left(S_{n}\right)$, since $f$ and $g$ are. Hence, since $G^{0}\left(S_{n}\right)=G^{I}\left(S_{n}\right)=Q\left(F_{0}\right)$, we have, by the definition of $Q\left(F_{0}\right)$, that $k^{-1} h^{-1} r f^{-1} r^{-1} g^{-1} h \mid B_{0}=\left(k^{-1} h^{-1} r \mid B_{0}\right)\left(f^{-1} r^{-1} \mid B_{0}\right)\left(g^{-1} h \mid B_{0}\right)$ is in $G^{0}\left(B_{0}\right)$. Since, 
as we showed above, $\left(f^{-1} r^{-1} \mid B_{0}\right)\left(g^{-1} h \mid B_{0}\right)$ is in $G^{0}\left(B_{0}\right)$, we have $k^{-1} h^{-1} r \mid B_{0}$ is in $G^{0}\left(B_{0}\right)$, since $G^{0}\left(B_{0}\right)$ is a group. Thus $h^{-1} r$ is in $Q\left(F_{0}\right)=G^{I}\left(S_{n}\right)=G^{0}\left(S_{n}\right)$, as was to be shown.

The group $G^{0}\left(S_{n}\right)$ is, as we have seen, a proper normal subgroup of $G\left(S_{n}\right)$. If $N$ is any proper normal subgroup of $G\left(S_{n}\right)$, then $G^{0}\left(S_{n}\right) \subset N$ by Theorem 3 . We have shown that $G^{0}\left(S_{n}\right)$ is of index 2 in $G\left(S_{n}\right)$ for $n \leqq 3$. Therefore, by fact (2) about groups of index 2 noted above, $G^{0}\left(S_{n}\right)=N$.

THEOREM 15. If $n \leqq 3$, the following subgroups of $G\left(S_{n}\right)$ are simple, open and closed in the topological group $G\left(S_{n}\right)$, and equal to one another:

(1) the group $D\left(S_{n}\right)$ of deformations of $S_{n}$;

(2) the group $H\left(S_{n}\right)$ of all homeomorphisms of $S_{n}$ which are homotopic to the identity $e$;

(3) the group $B\left(S_{n}\right)$ of homeomorphisms of $S_{n}$ of Brouwer degree 1;

(4) the identity component $C_{0}\left(S_{n}\right)$ of $G\left(S_{n}\right)$;

(5) the group of homeomorphisms $G^{0}\left(S_{n}\right)=G^{I}\left(S_{n}\right)$.

Proof. By Theorem 14, $G^{0}\left(S_{n}\right)=G^{I}\left(S_{n}\right)$ is a proper normal subgroup of $G\left(S_{n}\right)$. By Theorems 7 and $9, C_{e}\left(S_{n}\right)=D\left(S_{n}\right)=G^{0}\left(S_{n}\right)$, and this group is simple and open (and closed since $C_{e}\left(S_{n}\right)$ is closed). Since $S_{n}$ admits a homeomorphism $r$ of degree $-1, B\left(S_{n}\right)$ is a proper normal subgroup of $G\left(S_{n}\right)$. Since degree $(e)=1, r$ is not homotopic to $e$, by a theorem of Brouwer [36] (now a standard theorem of homology theory; see [38], where it is an immediate consequence of Axiom 5). Hence $H\left(S_{n}\right)$ is a proper normal subgroup of $G\left(S_{n}\right)$ (that it is a normal subgroup is verified as in Theorem 4). Thus, by Theorem 14, $B\left(S_{n}\right)=H\left(S_{n}\right)=G^{0}\left(S_{n}\right)$.

THEOREM 16. If $n \leqq 3$, then two homeomorphisms $f$ and $g$ of $S_{n}$ are isotopic if and only if they are homotopic.

Proof. If $f$ is isotopic to $g$, then $f g^{-1}$ is homotopic to $e$, hence $f g^{-1}$ is isotopic to $e$ by Theorem 15 , hence $f$ is isotopic to $g$.

6. Preliminary results on the group $G^{I}(M)$.

Theorem 17. Let $M$ be a manifold, $\operatorname{dim} M \leqq 3$. The index of $G^{I}(M)$ in $G(M)$ is $\leqq 2$.

Proof. If $G^{I}(M)=G(M)$, then $G^{I}(M)$ is of index 1 in $G(M)$. If $G^{I}(M)$ $\neq G(M)$, take an $r$ not in $G^{I}(M)$. Then $r^{-1}$ is not in $G^{I}(M)$. Take any $h$ not in $G^{I}(M)$. Choose a pivot cell $F_{0}$ in $M$ (Theorem 10), and set $B_{0}=$ Bndy $F_{0}$. Now proceed exactly as in the induction step of the proof of Theorem 14 to show that $h^{-1} r$ is in $G^{I}(M)$; this is possible because Theorems 12 and 13 are for manifolds $M, \operatorname{dim} M \leqq 3$, rather than for spheres $S_{n}, n \leqq 3$. As in Theorem 14 , this shows that $G^{I}(M)$ is of index 2 in $G(M)$.

TheOREm 18. If $M$ is an orientable closed manifold, $\operatorname{dim} M \leqq 3$, and $M$ admits a homeomorphism of degree -1 , then $G^{I}(M)=B(M)$. That is, a homeo- 
morphism $h$ of $M$ is of degree 1 if and only if $h$ is a finite product, $h=h_{1} \cdots h_{k}$, of homeomorphisms $h_{i}$ such that $h_{i}$ is the identity inside a closed $n$-cell $F_{i}$ in $M$, $n=\operatorname{dim} M$.

Proof. It follows from the definition of degree as given by Brouwer [37]; (see also [38]) that any homeomorphism which is the identity inside an $n$-cell in $M, n=\operatorname{dim} M$, is of degree 1 . Hence $E^{I}(M) \subset B(M)$. Therefore, since $B(M)$ is a group (see $\S 5$ ), $G^{I}(M) \subset B(M)$. By hypothesis, $B(M) \neq G(M)$. Since $G^{I}(M)$ and $B(M)$ are both of index 2 in $G(M)$ (see $\$ 5$ ), and $G^{I}(M) \subset B(M)$, we have $G^{I}(M)=B(M)$.

Note. If $M$ is an orientable closed manifold, $\operatorname{dim} M \leqq 2$, then $M$ admits a homeomorphism of degree -1 ; this is easily seen by direct construction, using the classification of such manifolds into the 2-sphere and 2-sphere with handles. However, there are orientable closed 3-manifolds which do not admit a homeomorphism of degree -1 [39].

\section{BIBLIOGRAPHY}

1. J. Kister, Isotopies in 3-manifolds with boundaries. I, Amer. Math. Soc. Notices vol. 6 (1959) Abstract 563-3, p. 642.

2. M.-E. Hamstrom and E. Dyer, Regular mappings and the space of homeomorphisms on a 2-manifold, Duke Math. J. vol. 25 (1958) pp. 521-531.

3. - Regular mappings and the space of homeomorphisms on a 3-manifold, Amer. Math. Soc. Notices vol. 6 Part I (1959) Abstract 564-39, pp. 783-784.

4. H. Tietze, Über stetige abbildungen einer quadrätflache auf sich selbst, Rend. Circ. Mat. Palermo vol. 38 (1914) pp. 247-304.

5. H. Kneser, Die Deformationssätze der einfach zusammenhangenden Flächen, Math. Z. vol. 25 (1926) pp. 362-372.

6. R. Baer, Kurventypen auf Flächen, J. Reine Angew. Math. vol. 156 (1927) pp. 231-246.

7. - Isotopie von Kurven auf orientbaren, geschlossenen Flächen und ihr Zusammenhang mit der topologischen Deformation der Flächen, J. Reine Angew. Math. vol. 159 (1928) pp. 101-116.

8. J. Schreier and S. Ulam, Über topologischen Abbildungen der euklidischen Sphären, Fund. Math. vol. 23 (1934) pp. 102-118.

9. - Eine Bemerkung über die Gruppe der topologischen Abbildungen der Kreislinie auf sich selbst, Studia Math. vol. 5 (1934) pp. 155-159.

10. H. Kneser, Reguläre Kurvenscharen auf Ringfächen, Math. Ann. vol. 91 (1924) pp. 135154.

11. H. Poincaré, Mémoire sur les courbes définies par une equation differentielle, J. Math. Pures Appl. ser. 3 vol. 7 (1881) pp. 375-422; ser. 3 vol. 8 (1882) pp. 251-296; ser. 4 vol. 1 (1885) pp. 167-244.

12. S. Ulam and J. von Neumann, On the group of homeomorphisms of the surface of the sphere, Bull. Amer. Math. Soc. vol. 53 (1947) Abstract 283 p. 506.

13. N. J. Fine and G. E. Schweigert, On the group of homeomorphisms of an arc, Ann. of Math. vol. 62 (1955) pp. 237-253.

14. R. D. Anderson, The algebraic simplicity of certain groups of homeomorphisms, Amer. J. Math. vol. 80 (1958) pp. 955-963.

15. M. K. Fort, Jr., A proof that the group of homeomorphisms of the plane onto itself is locally arcwise connected, Proc. Amer. Math. Soc. vol. 1 (1950) pp. 59-62.

16. J. H. Roberts, Local arcwise connectivity in the space $H^{n}$ of homeomorphisms of $S^{n}$ onto 
itself, Summary of Lectures, Summer Institute on Set Theoretic Topology, Madison, Wisconsin, 1955, p. 100.

17. A. Schoenflies, Beitrage zur Theorie der Punktmengen. III, Math. Ann. vol. 62 (1906) pp. 286-328.

18. - Die Entwickelung der Lehre von den Punktmannigfaltigkeiten, Leipzig, B. G. Teubner, 1908.

19. B. v. Kerekjart6, Vorlesungen über Topologie, Berlin, Julius Springer, 1923.

20. L. Antoine, Sur l'homeomorphie de deux figures et de leurs voisinages, Math. Pures Appl. ser. 8 vol. 4 (1921) pp. 221-325.

21. J. W. Alexander, An example of a simply connected surface bounding a region which is not simply connected, Proc. Nat. Acad. Sci. U.S.A. vol. 10 (1924) pp. 8-10.

22. E. Artin and R. H. Fox, Some wild cells and spheres in three-dimensional space, Ann. of Math. vol. 49 (1948) pp. 979-990.

23. J. W. Alexander, On the subdivision of 3-space by a polyhedron, Proc. Nat. Acad. Sci. U.S.A. vol. 10 (1924) pp. 6-8.

24. W. Graeub, Die Semilineare Abbildungen, Sitzungsberichte der Heidelberger Akademie der Wissenschaften, Springer-Verlag, Heidelberg, 1950.

25. E. E. Moise, Affine structures in 3-manifolds, II. Positional properties of 2-spheres, Ann. of Math. vol. 55 (1952) pp. 172-176.

26. R. H. Bing, Locally tame sets are tame, Ann. of Math. vol. 59 (1954) pp. 145-157.

27. - Approximating surfaces with polyhedral ones, Ann. of Math. vol. 65 (1957) pp. $456-483$.

28. - An alternative proof that 3-manifolds can be triangulated, Ann. of Math. vol. 69 (1959) pp. 37-65.

29. E. E. Moise, Affine structures in 3-manifolds, V. The triangulation theorem and Hauptvermutung, Ann. of Math. vol. 56 (1952) pp. 96-114.

30. O. Veblen, On the deformation of an n-cell, Proc. Nat. Acad. Sci. U.S.A. vol. 3 (1917) pp. 654-656.

31. J. W. Alexander, On the deformation of an n-cell, Proc. Nat. Acad. Sci. U.S.A. vol. 9 (1923) pp. 406-407.

32. T. Rad6, Über den Begriff der Riemannschen Flächen, Acta Szeged. vol. 2 (1925) pp. 101121.

33. I. Gawehn, Über unberandete zweidimensionale Mannigfaltigkeiten, Math. Ann. vol. 98 (1927) pp. 321-354.

34. D. E. Sanderson, Isotopy in 3-manifolds, II. Fitting homeomorphisms by isotopy, Duke Math. J. vol. 26 (1959) pp. 387-396.

35. E. E. Moise, Affine structures in 3-manifolds, III. Tubular neighborhoods of linear graphs, Ann. of Math. vol. 55 (1952) pp. 203-214.

36. L. E. J. Brouwer, Über Abbildungen von Mannigfaltigkeiten, Math. Ann. vol. 71 (1912) pp. 97-115.

37. P. S. Alexandrov, Kombinatornaya Topologiya, vols. 1 and 2, 1947, trans. by H. Komm, Rochester, Graylock Press, 1956.

38. S. Eilenberg and N. Steenrod, Foundations of Algebraic Topology, Princeton, Princeton University Press, 1952. 828.

39. H. Seifert, Verschlingungsinvarianten, Sitzungsberichte Berlin Akad. (1933) pp. 811-

\section{Louisiana State University,} Baton Rouge, Louisiana

Princeton University, Princeton, New Jersey 